\title{
Louis Rougier et la « mentalité réaliste »
}

\section{Laurent Cesalli}

\section{(2) OpenEdition}

\section{Journals}

\section{Édition électronique}

URL : https://journals.openedition.org/philosophiascientiae/466

DOI : 10.4000/philosophiascientiae.466

ISSN : 1775-4283

Éditeur

Éditions Kimé

\section{Édition imprimée}

Date de publication : 1 novembre 2006

Pagination : 157-175

ISBN : 2-84174-408-6

ISSN : $1281-2463$

\section{Référence électronique}

Laurent Cesalli, « Louis Rougier et la « mentalité réaliste » », Philosophia Scientiæ [En ligne], 10-2 | 2006, mis en ligne le 09 juin 2011, consulté le 21 septembre 2021. URL : http://journals.openedition.org/ philosophiascientiae/466; DOI : https://doi.org/10.4000/philosophiascientiae.466 


\title{
Louis Rougier et la «mentalité réaliste»
}

\author{
Laurent Cesalli \\ Albert-Ludwigs-Universität Freiburg i.Br.
}

\section{Introduction}

La chose est bien connue : Rougier a fait de la mise en question radicale du réalisme l'un des axes centraux de sa réflexion de philosophe et d'historien. L'expression «mentalité réaliste », lestée d'une connotation peu flatteuse, fait l'objet de développements critiques dans certains des plus grands textes du penseur français, des Paralogismes du rationalisme (1920) à son livre plus tardif intitulé La métaphysique et le langage (1960), en passant par La scolastique et le thomisme (1925) et le Traité de la connaissance (1955). ${ }^{1}$

L'objet de la présente étude est le lien supposé exister entre réalisme d'un côté, et faillite philosophique de l'autre - lien que Rougier ne se fatigue pas de réaffirmer. Je tenterai de suivre le philosophe français dans l'analyse de ce qui est à ses yeux un cas paradigmatique de mésalliance entre ambitions philosophiques et présupposés métaphysiques, à savoir la scolastique - laquelle, en termes rougiériens, n'est autre que le thomisme. La petite touche que je voudrais apporter au tableau du «philosophe engagé » qu'était sans aucun doute Rougier s'articulera en deux temps. Dans le premier, je tâcherai d'expliciter la notion de «mentalité réaliste ». Dans le second, j'esquisserai un mouvement cher à Rougier lui-même, puisque j'essaierai de relativiser sa critique du réalisme afin

Philosophia Scientice, 10 (2), 2006, 157-175.

${ }^{1}$ [Rougier 1920b] ; [Rougier 1925a]; [Rougier 1955a]; [Rougier 1960a] ; cf. aussi [Rougier 1966]. Sur le lien organique entre les ouvrages de 1920, 1925 et 1955, cf. [Rougier 1955a, 9] (avertissement) : « Cet ouvrage contient la justification épistémologique et logique, procurée après-coup, des deux autres livres qui l'ont précédé : Les paralogismes du rationalisme. Essai sur la théorie de la connaissance (1920) et La scolastique et le thomisme (1925). Les trois livres constituent, en effet, une sorte de trilogie qui devrait se lire comme un tout ». L'ouvrage de 1955 reprend et développe les idées de [Rougier 1921a]. 
de reconsidérer, dans une perspective plus équilibrée, la thèse du supposé naufrage philosophique de la scolastique. ${ }^{2}$

\section{La «mentalité réaliste »}

Dans La scolastique et le thomisme, Rougier nous raconte une histoire et part d'un diagnostic. Commençons par le diagnostic : la scolastique est une faillite philosophique. Pourquoi? Afin d'en avoir une idée, il nous faut esquisser les grands traits de l'histoire en question. La scolastique telle que nous la présente Rougier est essentiellement la tentative malheureuse de résoudre un problème précis et qui lui est propre, celui de l'accord de la raison et de la foi. Or cette tentative est doublement vouée à l'échec.

\section{Les raisons formelle et matérielle de la faillite}

En effet, s'il n'y a rien à redire à la forme d'un problème de type 'accord de $A$ avec $B$ ', la possibilité même de sa solution présuppose que l'on soit au clair sur la nature de $A$ et de $B$ - en l'occurrence, et selon les termes de Rougier, sur la valeur, la nature et la portée respectives de la foi et de la raison. La première cause de l'échec de la scolastique est un vice de forme, car le problème d'un possible accord a été pour ainsi dire directement affronté par les scolastiques avant ou sans que ne soit posé celui de la nature de ses termes.

La seconde cause de l'échec est matérielle : les aléas des cheminements spirituels et intellectuels de l'être humain ont fait s'incarner puis se rencontrer et se confronter les figures abstraites de la foi et de la raison sous les formes respectives du christianisme et de l'aristotélisme latins du milieu du XIII ${ }^{\mathrm{e}}$ siècle. Il ne s'agit nullement là de la première rencontre de la raison et de la foi, et encore moins de la première confrontation entre christianisme et aristotélisme - la plus grande partie de la patristique peut en témoigner - et Rougier est loin de l'ignorer. Ce qu'il y a ici de particulier, en revanche, c'est la rencontre d'éléments textuels, scientifiques et institutionnels - le corpus aristotélicien « retrouvé »,

\footnotetext{
${ }^{2}$ La présente étude est à lire de manière complémentaire avec celle d'Alain de Libera, ici même (cf. pp.177-206). Lors du colloque de Genève, nos contributions ont fait l'objet d'une présentation conjointe, les cahiers des charges ayant été définis de la manière suivante : Alain de Libera commençait par poser le problème dans ses dimensions et historique et philosophique larges et je poursuivais par une présentation de la critique du réalisme opérée par Rougier.
} 
avec ses critères de scientificité et la coexistence surveillée, au sein de l'Université, d'une faculté de philosophie, celle des arts, et d'une faculté de théologie - rencontre qui a pu faire passer la question de l'accord entre raison et foi, entre aristotélisme et christianisme, du statut de problème à celui de programme philosophique. Toutefois, et l'on peut voir là une forme de malchance des scolastiques, il s'avère que les termes dont il s'agit précisément d'expliquer l'accord sont incompatibles entre eux : tournez-le et retournez-le dans tous les sens, nous dit à juste titre Rougier, Aristote ne vous fournira jamais la justification rationnelle de deux éléments centraux de la révélation : la création du monde et la contingence des créatures.

\section{L'expédient scolastique}

A moins que, et c'est là le pivot de l'histoire que nous conte Rougier, à moins que l'on ne procède au «baptême d'Aristote» et rende par là sa philosophie non seulement apte, mais carrément prédestinée à fonder en raison ce que la foi nous commande de croire. Ignorant le vice de forme qui vouait son programme à l'échec, contournant l'obstacle matériel qui se présentait, la scolastique pouvait, en la personne de Thomas d'Aquin, son champion incontesté, commencer son œuvre de synthèse. Accusé de faux et d'usage de faux pour ce qui est de sa lecture d'Aristote, Thomas n'en est pas pour autant dépeint comme un mystificateur pervers. Il apparaît bien plutôt comme étant souffrant, paradoxalement atteint d'un mal philosophique contracté à la lecture d'Aristote lui-même, mal qui n'est autre que la mentalité réaliste. ${ }^{3}$

\section{Nature de la mentalité réaliste}

Les premières lignes du chapitre 4 de la deuxième partie du premier livre de La scolastique et le thomisme donnent le ton. Il vaut la peine de les lire intégralement :

« A lire les interminables discussions des scolastiques au sujet de la distinction réelle, formelle, virtuelle, modale ou de pure raison, de l'essence et l'existence, de l'essence ou de

\footnotetext{
${ }^{3}$ On voit ici s'esquisser un double paradoxe : non seulement l'aristotélisme est foncièrement incompatible avec la révélation dont on lui demande de fournir la justification rationnelle, mais la lecture erronée d'Aristote résulte d'une maladie philosophique qui a elle-même son origine chez le Stagirite.
} 
l'existence et de la subsistance, de l'esse in et de l'esse ad des relations, etc., on se convainc que tous les syllogismes mis en forme, toutes les distinctions considérées, toutes les formalités et les modalités introduites n'ont de signification qu'en vertu d'un certain type de mentalité, foncièrement distincte de celle que nous a façonnée la pratique des disciplines scientifiques, et que nous appellerons, faute de mieux, la mentalité ontologique ou réaliste. Décomposer les termes du langage, disséquer les notions de l'esprit en une série de concepts partiels, dont la démarcation est si ténue qu'on la perçoit à peine; hypostasier ces concepts, c'est-à-dire soutenir qu'à ces concepts, dissociés les uns des autres par une analyse toute mentale, correspondent adéquatement autant de réalités distinctes concourant à former le composé ontologique que la notion complexe représente; morceler, en conséquence, les êtres que nous dévoile la perception sensible ou la Révélation, en une série d'entités partielles, emboîtées les unes dans les autres, ou juxtaposées les unes à côté des autres, ou contractées en un tout composite, tels sont les procédés de cette mentalité particulière » [Rougier 1925a, 161].

Le mal est clairement identifié. Il s'agit d'une mentalité ontologique dont les symptômes sont des procédés projectifs allant toujours du linguistique ou du mental vers le réel, leur trait commun étant une tendance à hypostasier systématiquement tout élément conceptuellement circonscrit. Bref, le réaliste prend ses désirs linguistiques pour des réalités extramentales. L'étiologie de la mentalité réaliste est donnée sous la forme d'une liste de sept «principes du réalisme ontologique », eux-mêmes tempérés par cinq «principes restrictifs du réalisme ». Par souci de brièveté, je ne m'arrêterai que sur trois des-dits principes et leurs correctifs.

\section{Les principes constitutifs du réalisme}

Les trois principes fondamentaux du réalisme sont les suivants :

(1)[le principe essentiel du réalisme veut qu'] à toute notion distincte dans l'esprit corresponde adéquatement, hors de lui, une réalité objective qui contient formellement tout ce qui est inclus dans la définition de cette notion.

Appliqué à (1), le principe de raison suffisante - toute chose a une raison en vertu de laquelle elle est ce qu'elle est — donne lieu aux deux principes 
dérivés et corrélatifs suivants : le principe d'appartenance essentielle et le principe réaliste d'exclusion. Le premier exprime l'idée d'a-séité, le second celle d'ab-aliéité :

(2) toute chose possède par elle-même et immédiatement tout ce qui est inclus dans la définition de sa notion;

(3) tout ce qui n'est pas inclus dans la définition de la notion d'une chose ne lui appartient pas par elle-même, mais par autrui.

Le principe d'a-séité est le ressort des preuves ontologiques proposées par Anselme et Descartes - dans les deux cas, l'existence de Dieu est démontrée à partir du fait que l'existence est comprise dans la notion même de Dieu; le principe d'ab-aliéité permet quant à lui de distinguer, pour l'ensemble du créé, les questions portant sur l'essence - quid sit? - de celles portant sur l'existence — an sit? - et donc, en vertu de (1), de poser leur distinction réelle. En effet, puisque l'existence n'est incluse dans la définition d'aucune créature, il faut distinguer conceptuellement, pour toute créature, ce qu'elle est — quod est — de ce par quoi elle existe - quo est ${ }^{4}$; or, selon (1), ce qui peut être distingué en raison l'est également en fait; il s'ensuit que dans les créatures, l'essence est réellement distincte de l'existence.

\section{La « vérité fondamentale de la philosophie chrétienne »}

Ce point précis, cible privilégiée des critiques que Rougier adresse au thomisme, est selon lui l'acquis à la fois le plus important et le moins légitime de la mentalité réaliste à l'œuvre dans l'entreprise scolastique. Le plus important, parce qu'il s'agit, d'après le titre d'un article du Père Del Prado publié en mars 1910 dans la Revue thomiste, de la « vérité fondamentale de la philosophie chrétienne »[Prado 1910] : sans elle en effet, point de justification rationnelle des dogmes de la Trinité et de l'incarnation ${ }^{5}$; sans elle, la contingence du créé et la nécessité du Créateur se trouveraient dépourvues de tout fondement rationnel. Le moins légitime, parce qu'il est présenté, sur la base d'un passage des Seconds analytiques

\footnotetext{
${ }^{4}$ Cf. Boèce, De hebdomadibus, règles 2 à 8 . Dans ce texte, il y a un glissement de la distinction entre esse et id quod est (règle 2) à la distinction entre quod est et quo est (règles 7 et 8), cf. [Libera \& Michon (éds.) 1996, 30-32].

${ }^{5}$ [Rougier 1925a, 128-133]. C'est ce que Rougier appelle « le rôle organique joué dans le Thomisme par la distinction réelle de l'essence et de l'existence » [Rougier 1925a, 128].
} 
(II.7, 92b10) comme authentiquement aristotélicien alors que cette prétention ne peut tenir qu'au prix de la métamorphose opportune d'une distinction logique en une différence ontologique : Aristote se borne à dire qu'il est différent de savoir ce qu'est une chose et de savoir si cette chose existe tout en ajoutant immédiatement après (92b20) qu'une définition n'implique jamais l'existence de ce qu'elle définit. Thomas prend le premier passage comme antécédent pour en tirer une tout autre conclusion, à savoir le deuxième principe fondamental du réalisme évoqué ci-dessus, le principe d'appartenance essentielle.

Le lien assurément légitime entre thomisme et philosophie chrétienne, pour autant que l'on accepte cette dernière notion, résulte du processus d'élection du thomisme comme doctrine philosophique officielle de l'Eglise, processus que l'on peut juger achevé par l'encyclique Aeterni Patris promulguée par Léon XIII en 1879 et confirmée par Benoît XV quarante-deux ans plus tard avec Fausto appetente die, où l'on peut lire que l'Ordre des Prêcheurs s'est vu accorder la louange suprême lorsque l'Eglise a proclamé que la doctrine de saint Thomas était la sienne propre ( «cum Thomae doctrinam Ecclesia suam propriam edixit esse »). ${ }^{6}$

\section{Les principes limitatifs du réalisme}

Appliqués aveuglément, les principes fondamentaux du réalisme s'avèrent contre-productifs pour la scolastique. De fait, explique Rougier, ils conduisent à des hérésies, comme par exemple au panthéisme - une réification indifférenciée de l'être abolit la différence entre créateur et créatures - ou à des absurdités, par exemple au monisme des Eléates — une lecture « réifiante» de l'énoncé 'l'être est' donnant un tour de clé supplémentaire à la serrure de la prison parménidienne.

Rougier dénombre cinq facteurs rectificatifs du réalisme, dont voici les trois principaux : la théorie des Catégories tout d'abord, laquelle évite la réduction de l'être à un genre unique; la thèse du caractère transcendantal et analogique de l'être ensuite - l'être se dit aussi bien de la substance que des neuf accidents, mais en des sens différents et se trouvant dans un certain rapport les uns avec les autres; enfin, cette autre échappatoire au piège de Parménide qu'est la distinction entre

\footnotetext{
${ }^{6}$ Rougier se plaît à rappeler qu'au Concile de Trente, à côté de la Bible et des décrets pontificaux, la Somme de théologie trônait sur l'autel. Le processus en question commence avec l'acceptation de la doctrine thomasienne de l'âme rationnelle comme forme substantielle du corps par le Concile de Vienne (1312) et connaît un premier sommet avec la canonisation de Thomas par Jean XXII en 1323 [Rougier 1925a, 118].
} 
l'être en acte et l'être en puissance, puisqu'elle permet de rendre compte sans contradiction du devenir et du changement.

\section{L'origine aristotélicienne des principes du réalisme}

Le tableau brossé par Rougier n'est pas exempt de zones d'ombre. S'il est évident que le thomisme tel qu'il nous est présenté offre une instanciation parfaite de la mentalité réaliste, le rôle exact joué par Aristote demande à être précisé. Lorsqu'il se demande quelles sont « les raisons positives de l'adoption de l'aristotélisme par Thomas » [Rougier 1925a, 180], Rougier donne cette réponse générale : «dans l'œuvre du Stagirite se trouvent plus ou moins explicitement formulés les principes du réalisme ontologique », plus encore : « en enseignant la distinction logique de l'essence et de l'existence, Aristote inclinait les scolastiques à la transformer subrepticement en une distinction réelle »[Rougier 1925a, 181].

Aristote aurait donc élaboré la totalité des principes (constitutifs et restrictifs) du réalisme ontologique à l'exception d'un seul, qui n'est en réalité qu'une distinction logique, mais que tout, chez lui, inciterait à comprendre comme une différence ontologique. Rougier ne dit-il pas, à propos de la critique aristotélicienne des Idées, que dans sa tentative d'échapper à la métaphysique platonicienne, le Stagirite est tombé de Charybde en Scylla [Rougier 1960a, 62] ? Il ne s'agit pas ici de « disculper» Thomas, si tant est qu'il faille le faire, mais seulement de prendre en considération le fait que si Aristote est le véritable père du réalisme ontologique, le thomisme attaqué par Rougier ne peut être trop rapidement accusé d'hétérodoxie philosophique.

\section{Syntaxe grecque et mentalité réaliste}

L'étiologie de la mentalité réaliste ne s'arrête d'ailleurs pas à Aristote, ni même à Platon ou à Parménide. Sa véritable origine est pour ainsi dire impersonnelle, puisqu'elle est à chercher, comme nous l'explique Rougier dans les premiers chapitres de La métaphysique et le langage, dans certaines propriétés syntaxiques de la langue grecque car, ditil, « chaque système syntaxique est gros d'une métaphysique latente » [Rougier 1960a, 44]. Les deux malheurs de la syntaxe grecque sont la réduction de tout énoncé à la forme apophantique 'sujet-copule-prédicat' 
et l'introduction de l'article défini, lequel est responsable des «mésaventures idéologiques » [Rougier 1960a, 49] de ce qu'Aurélien Sauvageot, cité ici par Rougier, appelait les « civilisations de l'article $»{ }^{7}$ Non seulement Aristote, mais aussi les médiévaux, Leibniz, Kant et un grand nombre de logiciens contemporains, nous dit Rougier, ont été victime de l'hégémonie de la forme propositionnelle apophantique. Cette erreur logique n'est pas propre au réalisme, mais elle y produit des conséquences plus désastreuses qu'ailleurs. En effet : si tout ce qui peut s'énoncer est de forme ' $S$ est $P$ ' et si tout ce qui est conceptuellement distinguable est réellement distinct, alors l'acceptation d'une telle forme canonique du jugement implique une inflation ontologique galopante : l'analyse logicoontologique de la proposition 'cet arbre est vert' est d'(au moins) un tiers

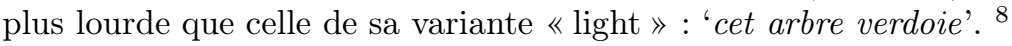

\section{Mentalité naturelle, mentalité primitive et mentalité réaliste}

Le choix des mots n'est jamais innocent : ce dont souffre la scolastique ne se résume pas à une thèse, ni même à un ensemble de thèses philosophiques, mais il s'agit d'une mentalité, c'est-à-dire d'une attitude ou d'un état d'esprit dont la portée déborde largement le domaine de la philosophie. Tout porte à croire que la notion de mentalité réaliste fait écho à celle de mentalité primitive élaborée dès 1910 par Lucien LévyBruhl. En 1922, soit deux ans après les Paralogismes du rationalisme et trois ans avant La scolastique et le thomisme, le père de la psychologie collective publie en effet un livre intitulé La mentalité primitive [Lévy-Brühl 1922]. A cette mentalité «mystique» ou «prélogique des primitifs », il oppose la mentalité logique de l'homme civilisé. Il n'en

\footnotetext{
${ }^{7}[$ Rougier 1960a, 49]. Notons que de ce point de vue, Rougier associe étroitement l'allemand au grec [Rougier 1960a, 48].

${ }^{8}$ Voir toutefois la critique de P.T. Geach, J. Barnes et L.-M. De Rijk à propos de la thèse selon laquelle la forme canonique du jugement chez Aristote est précisément ' $S$ est $P$ '. Ainsi, pour Aristote, la structure profonde de l'énoncé 'Socrate est blanc' estelle : '* [Sujet / attribut] ' (où '* ' représente un foncteur assertif monadique que l'on pourrait exprimer par 'il est le cas que') et non pas : '[Sujet / copule / attribut]'. [Rijk 1987, 53], [Rijk 2002, vol. I, 83-93]. [Barnes 1996, 187] : « The sentence 'Horses sleep' is a perfectly good categorical sentence : it predicates one term of another ; in it, sleep is said of horses, A is predicated of B. What are the terms in the sentence? 'Horses' and 'sleep'. What is left? Nothing. Where, then, is the copula? There is no copula; nor need there be»; c'est aussi une position défendue par P.T. Geach : « Aristotle neither had nor needed any theory of the copula; a proposition just consisted of a subject and a predidcate » [Geach 1972, 55].
} 
faut guère davantage pour poser que les scolastiques et les thomistes, et avec eux les innombrables victimes de la mentalité réaliste, sont les «sauvages » de la philosophie. Rougier ne le dit certes pas explicitement, mais ses propos n'interdisent pas qu'on le pense.

Au cours de son analyse, l'auteur de La scolastique et le thomisme, faisant écho à Bergson, pose la question de la légitimité de la mentalité réaliste en termes extrêmement généraux : « la mentalité réaliste est-elle la mentalité naturelle de l'esprit humain $? \gg .^{9}$ Il est étonnant que Rougier soulève ce problème que l'on serait tenté de qualifier, selon une construction à laquelle il a souvent recours lui-même, de «pseudoproblème », puisque tout relativiste sait qu'il ne peut y avoir quelque chose comme $l a$ mentalité naturelle de l'esprit humain. ${ }^{10}$ Rougier n'en donne pas moins une réponse remarquable à cette question : «qu'elle le soit de fait n'implique pas qu'elle le soit de droit » [Rougier 1925a, 183]. Cette discussion délimite également le terrain où se rencontrent le primitif ou encore l'enfant — c'est-à-dire l'humain avant l'âge de raison - et le réaliste : «le primitif et l'enfant prennent spontanément leurs rêves et leurs hallucinations pour des perceptions vraies » [Rougier 1925a, 183]. Plus techniquement, la mentalité primitive se caractérise par une confusion des quatre niveaux de langage que sont les discours logique, physique, psychologique et moral, ce qui a comme conséquence désagréable que le réaliste fait en réalité de la poésie, alors qu'il est convaincu de philosopher [Rougier 1960a, 30]. Pour achever le tableau, l'association entre réalisme et mentalité primitive met à jour une thèse anthropologique inacceptable et dont il ne nous appartient évidemment pas de discuter ici, thèse exprimée on ne peut plus clairement par Lucien Lévy-Bruhl au chapitre 14 de La mentalité primitive :

《Les primitifs procèdent par intuition directe, appréhension immédiate, interprétation rapide et presque instantanée de ce qui est perçu : il s'agit, par exemple, de lire sur le visage d'un homme des sentiments que peut-être il ne s'avoue pas à lui-même, de trouver les mots qui font vibrer la corde secrète que l'on veut toucher, de saisir le ridicule d'un acte ou d'une situation, etc. Ils sont guidés là par une sorte de

\footnotetext{
${ }^{9}$ [Rougier 1925a, 183]. Cf. [Bergson 1907, 352].

${ }^{10}$ Citons, pour illustrer le relativisme de Rougier, ce passage des Paralogismes $d u$ rationalisme : «Le Rationalisme admet l'unité de l'esprit humain, l'universalité et l'identité de la raison, entière en un chacun, dans le temps et dans l'espace. [...]. Il a fallu le renouveau des sciences historiques, au temps du Romantisme, qui marque un retour au sens de l'individuel et du concret, pour ruiner le dogme de l'identité de l'esprit humain » [Rougier 1920b, 455].
} 
flair ou de tact. L'expérience le développe et l'affine, et il peut devenir infaillible, sans avoir rien de commun avec des opérations intellectuelles proprement dites [...] L'observateur qui estimait hier l'intelligence du primitif virtuellement égale à celle de tout autre homme, le taxera aujourd'hui d'incroyable stupidité en le voyant incapable du raisonnement le plus simple $\gg .^{11}$

Que, dans ce texte, l'on remplace 'primitif' par 'réaliste', et l'on aura une idée de l'estime en laquelle Rougier tient les scolastiques. La mentalité réaliste, donc, maladie infantile de l'esprit humain, est aussi une maladie génétique de l'espèce. Elle fut combattue avec un succès inégal et l'histoire de la philosophie montre deux poussées notoires du mal au cours des siècles : l'aristotélisme original ${ }^{12}$ et sa reprise médiévale moyennant quelques retouches opportunes pour les besoins de la cause apologétique chrétienne.

\section{Rationalisme, mentalité réaliste et fiction démocratique}

Ajoutons qu'à côté de ses dimensions philosophique et anthropologique, Rougier semble prêter à sa critique du réalisme une dimension politique, comme en témoigne cette page dramatique de la préface au Traité de la connaissance :

« La révolution [...] due au développement de la pensée scientifique, a procédé ainsi à une véritable nuit du 4 août, en substituant à la conception monarchique de notions privilégiées, de principes s'imposant à notre esprit par droit divin et suivant un ordre hiérarchique par degré d'éminence, la conception démocratique de symboles, de conventions <et $>$ d'hypothèses librement choisies [...] » [Rougier 1955a, 19].

Toutefois, l'analogie ne vaut qu'à moitié : de fait, en plus d'avoir accouché de la mentalité réaliste, l'un des effets pervers du rationalisme universaliste est la «fiction» ou le «mythe» de l'égalité des compétences

\footnotetext{
${ }^{11}$ [Benda 1945, 213 et suiv.]. L. Lévy-Bruhl, dont on sait qu'il abandonnera ces idées par la suite, est aussi l'auteur d'un traité sur Les fonctions mentales dans les sociétés inférieures (1910) [Lévy-Brühl 1910].

${ }^{12}$ Sur le caractère naturel du réalisme d'Aristote, cf. [Rougier 1925a, 185] : « On peut donc avouer, en fait, dans l'Aristotélisme, ce que reconnaissait y être "La métaphysique naturelle de l'intelligence humaine" », cf. [Bergson 1907, 352].
} 
(directement dérivé de l'égale distribution de la raison chez tous les humains) entre citoyens, d'où l'idée erronée d'une compétence collective en matière législative critiquée par Rougier (par exemple dans Les Paralogismes du rationalisme, p. 48 et suivantes). Rougier n'en est pas un défenseur de l'Ancien Régime pour autant. Il ne condamne pas en ellesmêmes la Révolution ou la Déclaration universelle des droits de l'homme, mais il propose de substituer «au principe des majorités issu du rationalisme [...] le principe de la compétence [entendons : non-collective] issu de la sociologie positive [...]» (ibid.). La forme de gouvernement prônée par Rougier est une oligarchie des compétences (p. 52).

L'objet du Traité de la connaissance n'est certes pas de critiquer la pensée scolastique, mais d'évaluer le passage de l' «ancienne» à la «nouvelle théorie de la connaissance $» .{ }^{13}$ Toutefois, comme l'indique l'auteur lui-même, cet ouvrage fournit comme « après-coup » la justification épistémologique des Paralogismes du rationalisme et de La scolastique et le thomisme, les trois ouvrages constituant « une sorte de trilogie qui devrait se lire comme un tout $\gg .{ }^{14}$ Suivons donc ce conseil et voyons brièvement quel lien organique unit rationalisme, paralogismes, « ancienne théorie de la connaissance » et mentalité réaliste.

En deux mots : les principes constitutifs de la mentalité réaliste énumérés ci-dessus sont l'expression d'un paralogisme propre au rationalisme et que Rougier nomme «le paralogisme ontologique ». Celui-ci consiste à transgresser le principe selon lequel une définition n'implique jamais l'existence de ce qu'elle définit. On est victime du paralogisme ontologique lorsque l'on « fait jouer à une définition nominale le rôle d'une définition réelle $[\ldots]$ comme si elle créait son objet $\gg .{ }^{15}$ C'est par ce « passage illégal du concept à la chose » que l'on arrive à l'essence même du rationalisme, à savoir à la thèse de l'universalité de la raison humaine et de ses objets ou, pour reprendre les termes exacts de Rougier, à la croyance en « des vérités a priori, inconditionnellement nécessaires, in-

\footnotetext{
${ }^{13}$ Cf. [Rougier 1955a, 310]. Pour les « anciens » (i.e. les scolastiques et les rationalistes) les vérités non-empiriques, c'est-à-dire a priori, étaient fondées sur d'autre vérités universelles dites en soi, et non pas, comme c'est le cas dans la nouvelle théorie de la connaissance, sur des « conventions cohérentes <et > librement choisies ».

${ }^{14} \mathrm{Cf}$. [Rougier 1955a, 9] (texte cité ci-dessus, n. 1).

${ }^{15}$ [Rougier 1920b, 146]. Il faut relever avec Rougier que le principe auquel contrevient le paralogisme ontologique est aristotélicien et que ce paralogisme est explicitement dénoncé par Aristote dans les Réfutations sophistiques (5, 167a1-2, cf. aussi De interpretatione 11, 21a25-26). Aristote étant lui-même un cas exemplaire de réalisme (cf. [Rougier 1920b, 368-388]), on est tenté de se demander si Aristote a d'une certaine manière exploité un paralogisme qu'il a lui-même dénoncé $[. .$.
} 
dépendantes de notre esprit et de l'expérience $\gg .{ }^{16} \mathrm{Il}$ en résulte qu'être réaliste consiste précisément à être un rationaliste conséquent, à suivre l'inclination naturelle de l'esprit humain qui conduit à faire du paralogisme ontologique le principe fondamental de la philosophie. Historiquement, l'argument ontologique d'Anselme et la distinction réelle entre l'essence et l'existence sont deux manifestations extrêmes de cet état d'esprit.

\section{Les stigmates de la faillite : impasses et pseudo- problèmes}

La scolastique - ou le thomisme - est une faillite philosophique parce qu'il s'agit d'une pensée fondée sur un paralogisme. Selon Rougier, cet état de fait conduit à des impasses philosophiques et à la prise au sérieux de pseudo-problèmes. D'une part, la distinction réelle entre l'essence et l'existence, « vérité fondamentale de la philosophie chrétienne », conduit à des impasses philosophiques, comme l'avaient déjà relevé certains des critiques médiévaux de Thomas parmi lesquels ont peut nommer Siger de Brabant, Godefroid de Fontaines, Henri de Gand, Thierry de Freiberg, Jean Duns Scot ou encore Guillaume d'Ockham. Leurs objections ont la forme générale suivante : si l'on part du principe que l'essence est réellement distincte de l'existence, il devient difficile, voire impossible, d'expliquer (a) en quoi peut bien consister la réalité de l'essence en-dehors de l'existence, et (b) comment l'essence peut demeurer une puissance alors qu'elle n'est pas actualisée par l'existence. ${ }^{17}$

\footnotetext{
${ }^{16}[$ Rougier $1920 \mathrm{~b}, 1]$. La thèse de l'universalité de la raison humaine - c.-à.-d. de son égale distribution parmi les individus de l'espèce — résulterait ainsi d'une lecture « réalisante» de la définition de l'être humain comme animal doué de raison. De même, la thèse de l'existence de vérités a priori résulterait d'une interprétation 《existentielle » ou ontologique de la nécessité caractérisant les relations entre les termes de certaines propositions.

${ }^{17}$ [Rougier 1925a, 521 et suiv.; 600 et suiv.]. Duns Scot : Ord. II, d. 1, q.2; Rep. Par. II, d. 7, q. 2, n. 7 et $O p$. ox. II, d. 12, q. 1, n. 16. Ockham, Quodlibeta, II.7 et Summa logicae III.2, c. 27. Le principe général est ici le suivant : " tot sunt esse quot essentiae» (Henri de Gand et Godefroid de Fontaines). Pour un dossier complet de la question comprenant également des textes de Gilles de Rome et Jacques de Viterbe, cf. le Glossaire des sources donné par A. de Libera et C. Michon comme introduction aux traités de l'être et de l'essence de Thomas et de Thierry de Freiberg [Libera \& Michon (éds.) 1996, 15-36] ainsi que le dossier de textes [Libera \& Michon (éds.) 1996, 207-244]. Pour Siger, cf. l'argument a constantia subiecti invoqué contre Albert et Thomas pour nier la proposition ' homo est animal nullo homine existente' [Rougier 1925a, 570-573]. La thèse de la dépendance, pour la vérité d'une proposition, de l'existence en acte du sujet dont elle traite, également défendue avec véhémence
} 
D'autre part, l'adoption du 《réalisme nominal » (la croyance selon laquelle tout mot désigne une réalité à laquelle il est consubstantiellement lié) ${ }^{18}$ et celle du « réalisme conceptuel » (la confusion entre des notions de classes et des notions d'individus en raison de leur désignation par des noms substantifs) ${ }^{19}$ conduisent à voir des problèmes métaphysiques dans ce qui n'est en réalité que des questions de réglementation de l'usage de certains termes linguistiques. Le réalisme nominal incite par exemple à considérer que la forme du langage (étymologie, structure grammaticale) nous renseigne sur la nature de ce dont parle le langage, bref, c'est un déni du caractère conventionnel des langues. Le réalisme conceptuel est responsable quant à lui de la réification de l'universel ainsi que de la querelle qui en découle, mais aussi et surtout, de la distinction réelle entre essence et existence.

\section{La scolastique, une «faillite philosophique»}

J'ai annoncé dans mon introduction une relativisation de la critique à laquelle Rougier soumet la scolastique. Je la fonderai sur un examen des notions de scolastique, de thomisme et de réalisme. Cette prise de distance nous permettra, je l'espère, sinon de trancher, du moins de reconsidérer la question de savoir si la scolastique est une faillite philosophique, et si oui, en quoi exactement elle s'avère en être une.

La ligne générale de cette contre-critique s'inspire de la stratégie adoptée par Rougier lui-même lorsqu'il parle d'« indétermination du problème scolastique $\gg^{20}$ : de même que le problème de l'accord entre la raison et la foi suppose que l'on sache exactement ce que sont l'une et l'autre, de même, une critique du réalisme scolastique identifié au thomisme suppose que les objets dont il s'agit de montrer les défauts existent. Il semble pourtant que tel ne soit pas le cas simpliciter. En d'autres termes, il s'agit de dénoncer, puis de renoncer à une triple réduction : celle tout d'abord de «la » scolastique au problème de l'accord entre raison et foi; celle ensuite de la pensée de Thomas d'Aquin au 《 thomisme »; celle, enfin, « du » réalisme à sa forme la plus naïve incarnée dans ce que Rougier appelle le «paralogisme ontologique».

par Roger Bacon, sera condamnée en 1277 par Robert Kilwardby comme étant une erreur in logicalibus (cf. [Libera 2002a, ch. 2]).

${ }^{18} \mathrm{Cf}$. [Rougier 1960a, 31 et suiv.].

${ }^{19}$ Cf. [Rougier 1960a, 35 et suiv.].

${ }^{20} \mathrm{Cf}$. [Rougier 1925a, 102 et suiv.]. 


\section{« La » scolastique}

L'expression ' $l a$ scolastique' renvoie à une notion abstraite qui, si elle correspond à quelque chose dans l'histoire de la philosophie médiévale, évoque davantage une manière de travailler qu'un ensemble de thèses ou un programme philosophique. En termes rougiériens, qui parle de "la scolastique' se rend coupable de réalisme conceptuel. Qu'est-ce donc que la scolastique, s'il ne s'agit pas d'un courant de pensée? Comme l'écrit Marie-Dominique Chenu, la scolastique est une forme particulière du travail intellectuel — « penser est un métier»-dont les instruments privilégiés sont la grammaire et surtout la logique (ou dialectique) « appliquée à l'intelligence d'un texte, soit d'un texte suivi pour constituer un commentaire, soit de textes choisis comme base et preuve d'une construction spéculative. C'est là », précise Chenu, « dans sa structure mentale et littéraire, le trait décisif de la scolastique, en philosophie comme en théologie : forme rationnelle de pensée qui s'élabore consciemment et volontairement à partir d'un texte estimé comme faisant autorité » [Chenu 1954, 54 et suiv.]. Il n'est absolument pas question ici d'une pensée orientée vers la solution d'un unique problème, et encore moins du problème de l'accord entre la foi et la raison.

Prenons comme exemple Thomas lui-même dans le prologue de la Somme contre les Gentils. Il y est abondamment question de foi et de raison, de vérités démontrables et de vérités dépassant la raison humaine. Si le problème fondamental de la scolastique avait été d'établir l'accord entre foi et raison, nul doute que Thomas en aurait fait l'objectif central de ce traité. Or, dans le chapitre deux du prologue, il dévoile son intention en ces termes : « exposer la vérité que professe la foi catholique tout en réfutant les erreurs contraires ». Argumenter au sujet des vérités révélées, démontrer ce qui peut l'être et critiquer les arguments de ses adversaires, cela n'est pas vouloir établir l'accord entre raison et foi. Car non seulement l'établissement de cet accord n'est pas l'objectif central de Thomas dans la Somme - ni ailleurs - mais il est impossible qu'il le soit. En effet, dans la perspective thomasienne, il ne s'agit pas d'un problème, mais d'un fait, puisque, comme le dit l'auteur au chapitre sept du même prologue, les vérités établies par la raison humaine ne peuvent tout simplement pas être contraires à la Révélation. Inutile de bâtir toute une scolastique pour établir un fait qu'il suffit de constater [...]

Selon toute probabilité, le «problème fondamental de la scolastique » n'en était donc pas un pour le plus grand des scolastiques lui-même. Cela tient au fait - et c'est un autre point sur lequel il est difficile de suivre Rougier - que contrairement à ce que pense ce dernier, Thomas 
a soigneusement examiné la question de la valeur, de la nature et de la portée de la raison humaine et de la foi. Que l'on consulte, pour s'en convaincre, les prologues de la Somme théologique et du commentaire au De trinitate de Boèce où philosophie et théologie sont très précisément distinguées, tant sur le plan des principes que sur celui de la méthode. ${ }^{21} \mathrm{Il}$ en résulte que le problème pointé par Rougier dans la soi-disant impasse faite par les scolastiques sur les questions de la nature, de la portée et de la valeur respectives de la foi et de la raison est lui-même ce qu'il appellerait un pseudo-problème. Je me permets de vous renvoyer, à ce propos, à la déconstruction de l'arrière-plan de l'encyclique Fides et ratio à laquelle a procédé Alain de Libera dans son livre Raison et foi [Libera 2003].

\section{« Le » thomisme et l' « a-thomisme »}

La deuxième réduction à laquelle il s'agit de renoncer est double. Premièrement, ce n'est qu'au prix de ce que J. Jolivet a appelé une « synecdoque autoritaire » [Jolivet 1991, 12] — c.-à.-d. abusive — que l'on peut ramener la philosophie médiévale, et avec elle, la scolastique au thomisme. De fait, si l'on fait sienne l'acception méthodologique proposée par Chenu, la scolastique n'est réductible à aucune philosophie ou pensée particulière, précisément parce qu'il s'agit d'une notion formelle, vide de tout contenu philosophique.

Deuxièmement, il faut renoncer à réduire la pensée de Thomas au thomisme. Commençons par enfoncer deux portes que l'on voudrait ouvertes. D'une part, Thomas n'était pas thomiste - pas plus qu'Averroès n'était averroïste. De l'autre, Thomas ne savait pas qu'il était saint Thomas, ni que sa doctrine allait devenir celle de l'Eglise catholique. En revanche, les auteurs à travers lesquels Rougier voit la philosophie médiévale et la pensée de Thomas (Mandonnet, Garrigou-Lagrange, Del Prado) sont des thomistes et défendent la position privilégiée de Thomas comme penseur officiel de l'Eglise. Ces deux différences contribuent à expliquer le tableau réducteur et unilatéral que Rougier brosse de la pensée de Thomas.

\footnotetext{
${ }^{21}$ Deux textes de Thomas, pour confirmer cela : (1) Summa theologiae, I, 10, ad 2 «theologia quae ad sacram doctrinam pertinet, differt secundum genus ab illa theologia quae pars philosophiae ponitur » (c'est la différence entre la théologie et la métaphysique), à ce propos, cf. [Gilson 1920 (1922), 34]. (2) Responsio ad Johannem Vercellensem, a. 33 : « Nec video quid pertineat ad doctrinam fidei qualiter Philosophi verba exponantur », à ce propos, cf. [Cheneval \& Imbach 1993, LIX].
} 
La distinction réelle entre essence et existence peut bien être la vérité fondamentale de la philosophie chrétienne, et même du thomisme [...] Il est difficile de montrer qu'elle est la vérité fondamentale de la pensée de Thomas, ${ }^{22}$ sauf, bien entendu, si ce dernier est envisagé dans une perspective exclusivement apologétique. En ce sens, Rougier s'avère en définitive plus thomiste que les thomistes eux-mêmes. Bref, la position de Rougier face au thomisme - on pourrait parler ici de son «a-thomisme » - est dirigée contre un objet certes réel - le thomisme a existé et existe encore - mais qui ne peut pas être identifié à la pensée du philosophe du XIII ${ }^{\mathrm{e}}$ siècle à laquelle on a pris l'habitude de l'associer.

\section{«Le » réalisme}

Une bonne partie de la présente étude a consisté à reconstruire la notion rougiérienne de réalisme, pour ainsi dire de l'intérieur, c.-à.-d. en suivant ses analyses de la scolastique et de sa supposée essence philosophique. Si l'on en croit l'auteur de La scolastique et le thomisme, le réalisme est l'aboutissement logique de la rencontre entre rationalisme et tendance naturelle ou primitive de l'esprit humain. Le paralogisme ontologique, à savoir le passage illégal du concept à la chose, constitue son expression la plus pure. Or, s'il est sans doute légitime d'appeler 'réaliste' une pensée fondée sur le paralogisme ontologique, deux restrictions importantes doivent être apportées aux propos de Rougier : d'une part, il est erroné d'affirmer que le paralogisme en question est essentiel à la pensée de Thomas; de l'autre, si le Moyen Âge est peuplé de réalistes, il n'en est à ma connaissance aucun qui ait érigé ledit paralogisme en principe philosophique. ${ }^{23}$

\footnotetext{
${ }^{22}$ S'il semble que l'on puisse inférer du c. 4 du De ente et essentia que l'être et l'essence doivent être réellement distincts, la nature réelle de la distinction n'y est toutefois pas explicitement affirmée et ne fait donc pas l'objet de développements particuliers. La distinction réelle est plus souvent impliquée (à partir de la notion de composition réelle) qu'affirmée par les propos de Thomas. Pour des passages où elle se trouve explicitement affirmée, cf. le commentaire du De hebdomadibus, 2.32 ; In I Sent., d. 1, q. 13, a. 1, ad 3 et d. 19, q. 2, a. 2, ainsi que De veritate, q. 27, a. 1, ad 8. Sur la controverse (inaugurée par Siger de Brabant et qui dure aujourd'hui encore) au sujet de la nature de la distinction, cf. l'introduction, les notes et le dossier de textes dans [Libera \& Michons (éds.) 1996].

${ }^{23} \mathrm{~A}$ l'exception notable de l'argument ontologique d'Anselme puisqu'il est vrai que celui-ci peut être critiqué en retraçant dans sa preuve le paralogisme ontologique, mais il est également vrai que Thomas a critiqué l'argument d'Anselme précisément (Somme théologique, I, q. 2, a. 1) en raison du fait qu'il y a une différence (une distinction?) essentielle entre concevoir l'existence de $x$ et l'existence réelle de $x$. Il est intéressant de noter que Rougier relève lui-même ce fait [Rougier 1925a, 193-194].
} 
Si Thomas avait été un réaliste «à la Rougier», il aurait déduit les lois de l'être à partir de celles de la pensée, il aurait réduit la métaphysique à l'épistémologie ou à la psychologie, bref, il aurait été un idéaliste avant l'heure. Or il semble que Thomas, comme la plupart des scolastiques, ait suivi le principe rigoureusement inverse : ab nosse ad esse non valet consequentia. Tel est l'un des principes limitatifs de la théorie de la connaissance médiévale, si l'on en croit Etienne Gilson, auteur que l'on ne soupçonnera pas d'a-thomisme, dans ses deux opuscules Le réalisme méthodique et Le réalisme thomiste (1947). La révolution copernicienne a eu lieu non pas avec Kant, mais avec Descartes déjà, dont le cogito est la conséquence de la contradictoire : ab nosse ad esse valet consequentia. D'où le retournement rhétorique proposé par Gilson pour décrire l'orientation épistémologique fondamentale des médiévaux : «res sunt, ergo cogito » [Gilson 1935, 81 et suiv.].

Prenons comme exemple l'adage tiré du De sensu et sensato d'Aristote et jouissant d'une reconnaissance pratiquement universelle au Moyen Âge : nihil est in intellectu, quod prius non fuerit in sensu ${ }^{24}$ — il n'y a rien dans l'intellect qui ne fut auparavant dans les sens. C'est à mon avis la même idée qu'exprime Gilson dans Le réalisme méthodique (p. 79) nous mettant en garde contre la confusion entre un réalisme tirant son nom du fait qu'il est fondé dans les choses et un réalisme «magique » qui serait une forme extrême d'idéalisme : «le fait que toute existence me soit donnée dans une connaissance n'implique aucunement que ma connaissance soit la cause de cette existence ». Gilson et Rougier combattent un même ennemi, mais sous deux appellations différentes dont l'une est équivoque : d'une part, le passage illégal du concept à la chose s'appelle 'idéalisme' pour Gilson et 'réalisme' pour Rougier ; d'autre part, idéalisme et réalisme ne font qu'un pour Rougier, alors qu'ils s'opposent radicalement pour Gilson. Les deux penseurs contemporains tombent donc d'accord sur la dénomination de 'réaliste' appliquée à Thomas mais entendent par là des choses totalement différentes.

\section{Conclusion}

Une fois pondérées les notions de scolastique, de thomisme et de réalisme, tâchons de reconsidérer la question de savoir si, et si oui, en quoi, la scolastique est une faillite philosophique. Il est légitime de parler de faillite soit lorsqu'un objectif que l'on s'était fixé n'est pas atteint, soit lorsqu'il est atteint, mais de manière incorrecte. Autrement dit, on peut

${ }^{24}$ Cf. Aristote, De sensu et sensato, 445b16-17 ; voir aussi [Hamesse 1974, 197]. 
parler de faillite philosophique lorsqu'un objectif philosophique n'est pas atteint, ou alors lorsqu'il est atteint de manière non-philosophique.

Entendue en son sens non-réducteur - à savoir comme n'étant pas identifiée à un courant de pensée bien déterminé - la scolastique tend à élaborer des pensées originales nourries de différentes traditions philosophiques. Il est difficile de voir là un quelconque objectif philosophique. En revanche, la pensée des scolastiques est éminemment philosophique. Il suffit pour s'en convaincre de parcourir les traités, les questions et les sommes des auteurs médiévaux. Le tri entre arguments d'autorité et de raison est soigneusement opéré, et la démarche est d'une rigueur scientifique qui n'a souvent rien à envier aux grands textes de la philosophie analytique contemporaine. Dépourvue d'objectif philosophique propre, la scolastique entendue en son sens non-réducteur échappe au premier trait qui pourrait la stigmatiser comme étant une faillite. Procédant de manière philosophique par excellence, les scolastiques échappent également au second trait qui menace de faire de leur pensée une faillite philosophique.

Cela dit, voyons ce qu'il en est si l'on accepte la notion rougiérienne de scolastique. Partons donc du principe que la scolastique n'est rien d'autre que la philosophie de Thomas. Celui-ci a-t-il eu comme objectif d'accorder la foi et la raison? Etant donné le soin extrême que met l'Aquinate à tenir séparé ce qui relève de la foi et ce qui concerne la raison, on peut mettre en doute que montrer l'accord de ce que l'on s'efforce de tenir séparé puisse être un objectif philosophique raisonnable. Au contraire, comme nous l'avons vu à l'exemple du prologue de la Somme contre les gentils (ch. 7), il s'agit là d'un problème philosophiquement vidé de sa substance parce que sa solution est pour ainsi dire (déjà) donnée. Thomas ne s'est donc tout simplement pas donné l'objectif que Rougier lui reproche de ne pas avoir atteint.

Considérons un dernier cas de figure : admettons que l'accord entre foi et raison ait été l'objectif philosophique de Thomas et que celui-ci ait organisé sa pensée tout entière dans le seul but de l'atteindre. Sa démarche se laisserait-elle pour autant philosophiquement disqualifier comme étant non-philosophique? Nullement : il ne suit pas du fait que la distinction réelle entre essence et existence est une thèse philosophique critiquable qu'elle n'est pas le résultat d'une démarche authentiquement philosophique. On serait bien en peine de trouver cette perle rare : une thèse philosophique qui ne soit pas critiquable. On peut avoir tort et être cohérent. Tout dépend des principes que l'on aura admis au départ. 
L'histoire de la philosophie montre que tout philosophe à tort aux yeux d'au moins un autre philosophe, mais cela ne veut pas dire que tout philosophe procède de manière non-philosophique.

Bref, on peut penser que la distinction réelle est indéfendable, que le réalisme qui la sous-tend est philosophiquement critiquable et que, par suite, Thomas a tort sur ce point. Il en faut plus pour faire de sa pensée une faillite philosophique. Elle est tout au plus une faillite herméneutique pour qui verrait en la philosophie de Thomas une tentative de restituer le plus fidèlement possible la pensée d'Aristote. Mais là encore, ce serait réduire la pensée de l'Aquinate à une vaste entreprise d'explicitation de la doctrine du Stagirite, ce qu'elle n'est assurément pas.

En conclusion : si légitime soit-elle — personne ne veut d'une «ontologie de Schengen », d'une philosophie du libre passage du concept à la chose - la critique du réalisme menée par Rougier se trompe doublement d'objet : d'une part, la position philosophique caricaturale décrite par l'auteur de La scolastique et le thomisme sous l'appellation de 'mentalité réaliste' n'a pas (ou peu) de réalité dans l'histoire de la philosophie et, peut-être moins qu'ailleurs, dans celle de la philosophie médiévale; de l'autre, Thomas d'Aquin, s'il est vrai qu'il soutient la distinction réelle de l'essence et de l'existence, n'en fait pas, au contraire de ce que pouvaient suggérer certains de ses lecteurs du début du $\mathrm{XX}^{\mathrm{e}}$ siècle, le principe fondamental de sa pensée. 